\title{
Avascular Necrosis of Metacarpal Head in an Amateur Volleyball Player: A Case Report
}

\author{
Prahalad K Singhi ${ }^{1}$, Sivakumar Raju ${ }^{2}$, Venkatappa Somashekar ${ }^{3}$, Raghava Kumar ${ }^{4}$
}

\begin{abstract}
Avascular necrosis of the metacarpal head is very rare compared to that of femoral head, talus, or scaphoid. Mauclaire disease also known as Dietrich's disease is a rare condition that refers to osteonecrosis of the metacarpal head. It can be multifactorial, usually associated with systemic lupus erythematosus (SLE), steroid use, or trauma. We present a case of 13-year-old boy, an amateur volleyball player presented with pain and swelling of the right index finger for 3 months. Initial X-rays were normal but subsequent X-rays revealed a mild flattening of the second metacarpal head; blood investigations were normal. The MRI revealed osteonecrosis with mild flattening of the second metacarpal head with synovial effusion. The patient was misdiagnosed as tuberculosis of the metacarpal head before presenting to us. The patient was treated conservatively with good functional outcome. Though several cases of Dietrich's disease have been reported in the literature but none in a volleyball player, just to stress that repeated microtrauma could also be an etiological factor and a rare differential diagnosis in patients with the painful metacarpophalangeal (MCP) joint. Most of the patients can be managed conservatively with good functional outcome. Curettage and bone grafting, transfer of metatarsal head, osteotomies, arthroplasty (excision or prosthesis), or arthrodesis have been described as various surgical means of treatment. Keywords: Avascular necrosis, Metacarpal head, Volleyball player.

Journal of Orthopedics and Joint Surgery (2020): 10.5005/.jp-journals-10079-1020
\end{abstract}

\section{BACKGROUND}

Isolated avascular necrosis of the metacarpal head is a rare entity, first described by Mauclaire in 1927, but Maes et al. erroneously described this as the Dietrich's disease in 1932 due to obscured referencing. 'It has been associated with steroid use, systemic lupus patients, or following trauma. The spectrum of symptoms ranges from asymptomatic to severe pain and swelling, which may lead to a restricted range of motion of the metacarpophalangeal (MCP) joint.

\section{Case Description}

A 13-year-old amateur volleyball player presented with pain and swelling over second MCP for past 3 months (Fig. 1). The patient on initial consultation elsewhere was treated with analgesics and had a transient relief of pain but later aggravated as he continued to play volleyball. Initial X-ray was normal but one repeated at 3 months had some changes over second metacarpal head (Fig. 2) and he was suggested biopsy. Then, he presented to us with persistent pain; on clinical examination, the patient had an obvious swelling of the second MCP joint with joint line tenderness. There was no obvious asymmetry clinically and his flexion and extension range of movements were terminally painful.

Routine blood investigations, including complete blood count and renal profile, were normal. Markers of acute inflammation including C-reactive protein (CRP) and erythrocyte sedimentation rate (ESR) were within normal limits. Serology for rheumatoid factor, lupus, and antinuclear antibodies was also negative.

Radiographs revealed sclerosis and mild flattening of the head of the second metacarpal compared to previous radiograph done 3 months back, which arouse us to suspect avascular necrosis (AVN). The MRI of the right hand was done and revealed evidence of T1W low signal/T2 STIR bright signals with sclerosis involving right metacarpal head epiphysis representing AVN; minimal synovial effusion noticed in the MCP joint with coexisting chondrolysis of second metacarpal head was noticed (Fig. 3). Due to his good
${ }^{1-4}$ Department of Orthopedics, Preethi Hospitals Pvt. Ltd., Madurai, Tamil Nadu, India

Corresponding Author: Prahalad K Singhi, Department of Orthopedics, Preethi Hospitals Pvt. Ltd, Madurai, Tamil Nadu, India, Phone: +91 8122192632, e-mail: docpsin2001@yahoo.co.in

How to cite this article: Singhi PK, Raju S, SomashekarV, et al. Avascular Necrosis of Metacarpal Head in an Amateur Volleyball Player: A Case Report. J Orth Joint Surg 2020;2(1):46-48.

Source of support: Nil

Conflict of interest: None

functional status and range of movements, a conservative line of management was started. He was treated with rest and nonsteroidal

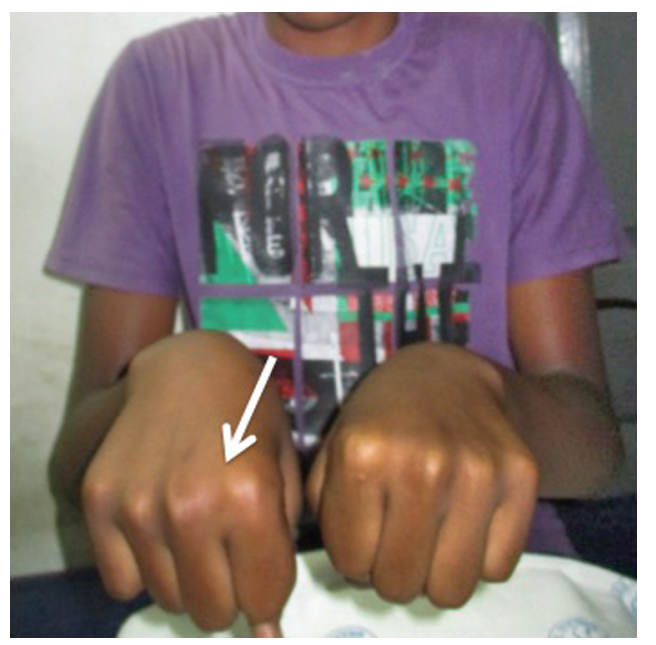

Fig. 1: Clinical examination of hand showing swelling of second metacarpophalangeal joint (arrow) 
anti-inflammatory medication. The patient was advised to abstain from involvement in active contact sports. At the end of 4 weeks, his pain had significantly reduced with improved visual analog score and after 2 years' follow-up there was no evidence of progression of disease or no asymmetry of metacarpal heads on flexion of fingers.

\section{Discussion}

Dietrich's disease is an uncommon condition affecting the metacarpal heads. Mauclaire from Paris had first described it in 1927 as "non-traumatic epiphysitis adolescentium" for a traumatic bilateral flattening of the metacarpal head in young boys. ${ }^{2}$

Although any of the fingers may be involved, long finger are more commonly involved compared to ring and little fingers, and thumb is rarely affected; male dominance was also described. Bimodal affection has been described in young adolescents (13-16 years) because of osteochondritis or adults over 45-50 due to trauma or steroid-induced. ${ }^{3}$ Flattening and collapse of the metacarpal head may be seen on plain-film radiography, although additional scintigraphy or MRI may be required for diagnosis. ${ }^{4}$

The pathologic changes are equivalent to those of avascular necrosis of other bones. There is disruption of critical blood supply leading to bone infarction, central necrosis, and surrounding hyperemia. Microfractures ensue, resulting in flattening and deformity of the bone surface. In cases of SLE, vasculitis of these
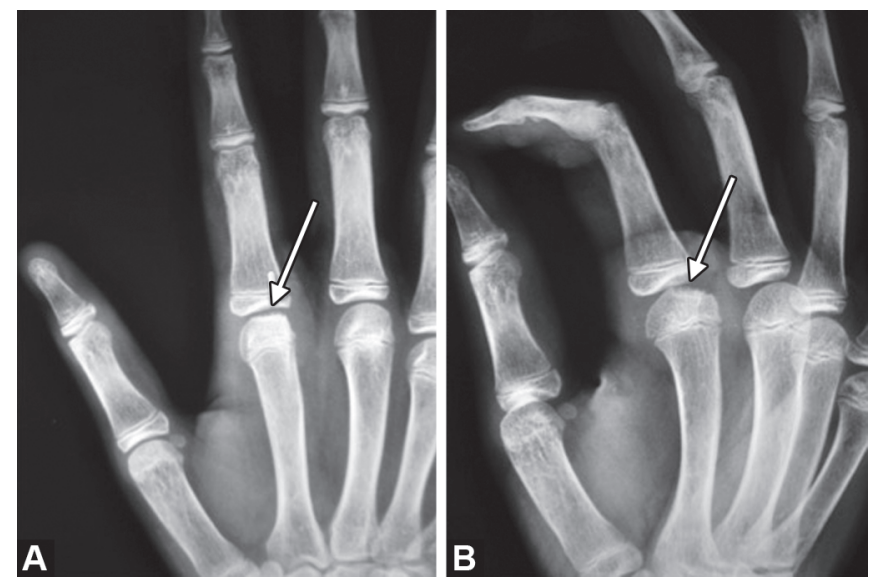

Figs $2 A$ and B: X-ray showing collapse of head of second metacarpal end vessels is thought to lead to necrosis. Repeated micro trauma, dermatomyositis, vasculitis may affect the load transmission on central pillar is also been described as etiological cause. ${ }^{5}$ In our case, with increased physical activity as an amateur volleyball player, an element of repetitive stress or microtrauma to his MCP joint may possibly have contributed to develop the condition.

This patient was planned for biopsy to rule out tuberculosis; so, that can be a differential diagnosis particularly in this part of the world. The MRI is still a very good noninvasive modality of diagnostic tool, which can differentiate both the conditions very well. The common differential diagnosis being traumatic arthritis, rheumatoid arthritis, infection, tubercular osteomyelitis, tenosynovitis of tendon sheath, and giant cell tumor of tendon sheath. ${ }^{6}$

Rest and use of nonsteroidal anti-inflammatory medications may be sufficient to control symptoms, and conservative treatment has been reported to be successful. ${ }^{7}$ When conservative treatment fails, surgery may be considered. ${ }^{8}$

Several surgical procedures have been described like osteochondral mosaicplasty for central and dorsal lesions. ${ }^{9}$ Flexion osteotomy of the metacarpal head (open wedge) can shift the articular surface if there is no dorsal lesion and the patient has painful crepitus. ${ }^{10}$ Transplantation of metatarsal head, ${ }^{11}$ Articular incongruity occurs when patient presents late in old age with pain, then arthroplasty is the best solution. Currently available designs (SRTM MCP implant) have shortcoming of luxations and difficult revisions since they are partially cemented but give good outcomes in terms of pain relief and range of motion. ${ }^{11}$

Whether this patient will be at an increased risk of early arthritic involvement of his MCP joint in the future remains to be seen. Nevertheless, his excellent functional status and resolution of pain demonstrate that conservative therapy can be successful. Surgery can be considered if symptoms return and become refractory to conservative measures. Long-term follow-up is also required to analyze for late shortening of the metacarpal, resulting in asymmetry in the metacarpal heads.

\section{Conclusion}

Dietrich's disease is a rare entity difficult to diagnose or easily missed. It should always be a diagnosis of exclusion for metacarpophalangeal joint pain particularly in patients with contact sports with repetitive
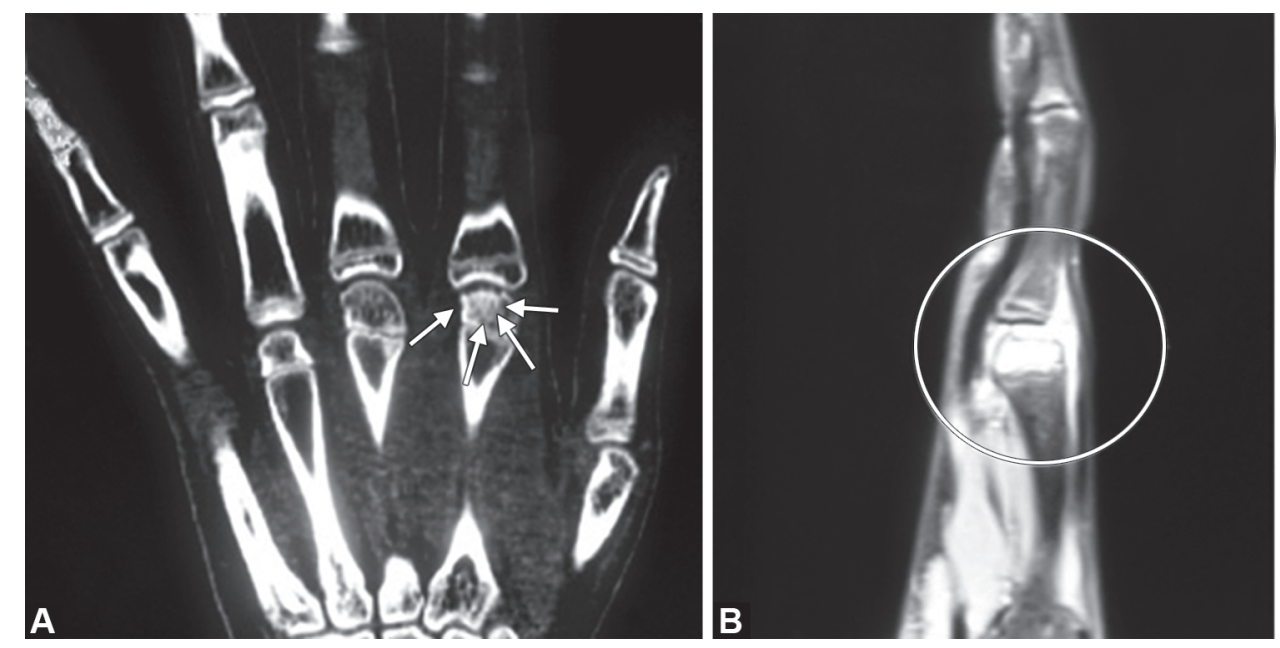

Figs $3 \mathrm{~A}$ and B: MRI revealing features of Dietrich's disease 
microtrauma. Most of them respond to the conservative line of treatment; surgical intervention is beneficial in exceptional cases.

\section{Acknowledgment}

Department of Orthopedics, Preethi Hospitals Pvt. Ltd., Madurai, Tamil Nadu, India: This manuscript was not presented as part or whole at any scientific meeting nor published anywhere earlier. This manuscript has been read by all the authors approved and represents honest work.

\section{References}

1. Mauclaire M. Epiphysite des tetes metacarpiennes avec main un peucreuse. Bull Mem Soc Nat Chir 1927;53:1377-1378.

2. GoffCW. Legg-Calve-Perthes syndrome and related osteochondroses of youth. Springfield, IL: Charles C. Thomas; 1954.

3. Weissman BN, Rappoport AS, Sosman JL, et al. Radiographic findings in the hands in patients with systemic lupus erythematosus. Radiology 1978;126(2):313-317. DOI: 10.1148/126.2.313.

4. Hu MH, Chen WC, Chang CH. Idiopathic osteonecrosis of the third metacarpal head. J Formos Med Assoc 2008;107(1):89-92. DOI: 10.1016/S0929-6646(08)60014-5.
5. Robinson $A B$, Rabinovich $C E$. Avascular necrosis of the metacarpals in juvenile dermatomyositis. J Clin Rheumatol 2010;16(5):233-236. DOI: 10.1097/RHU.0b013e3181e9345d.

6. De Smet L. Avascular necrosis of the metacarpal head. J Hand Surg Br 1998;23(4):552-554. DOI: 10.1016/S0266-7681(98)80149-6.

7. Wijeratna MD, Hopkinson-Woolley JA. Conservative management of Dieterich disease: case report. J Hand Surg Am 2012;37(4):807-810. DOI: 10.1016/j.jhsa.2011.12.018.

8. Reddy VM, Mathew VJ, Pancholi S, et al. Dieterich disease treated with curettage and bone grafting: A case report. J Orthop Case Rep 2018;8(2):13-15. DOI: 10.13107/jocr.2250-0685.1026.

9. Maes M, Hansen L, Cheyns P. Osteochondral mosaicplasty as a treatment method for bilateral avascular necrosis of the long finger metacarpal: Case report. J Hand Surg Am 2010;35(8):1264-1268. DOI: 10.1016/j.jhsa.2010.04.014.

10. Wada $M$, Toh S, Iwaya $D$, et al. Flexion osteotomy of the metacarpal neck: A treatment method for avascular necrosis of the head of the third metacarpal: A case report. J Bone Joint Surg Am 2002;84A(2):274-276. DOI: 10.2106/00004623-200202000-00016.

11. Schmidt I. The idiopathic avascular osteonecrosis of the third metacarpal head (M. Mauclaire/Dieterich's disease). Int J Case Rep Images 2017;8(2):92-95. DOI: 10.5348/ijcri-201714-CR-10753. 